\title{
Auf ein Wort: Zur kommunikativen Bedeutung des Wortes ueigentlich» in der Psychotherapie
}

«Eigentlich wollte ich gar nicht in die Therapie zu Ihnen kommen», sagt der Patient, und nun sitzt er doch hier. Was ist die Botschaft dieses Satzes? Drückt er die Einsicht aus, dass er der Therapie doch bedarf? Will der Patient sagen, er selbst habe keine Behandlungsmotivation und sei auf Drängen seiner Angehörigen hier? Das Wort «eigentlich» in dem Satz vernebelt den Kern der Aussage. «Eigentlich hat mir die Therapiestunde jetzt doch ganz gut getan.» Was will er uns damit sagen? Hat sie ihm gut getan oder ist er nur höflich? Warum sagt er nicht schlicht: «Das hat mir gut getan», sondern macht durch das «eigentlich» die Aussage unklarer?

Als Psychotherapeuten erleben wir recht häufig, dass Patienten durch bestimmte Formulierungen ihre Aussagen relativieren und unscharf machen. So drückt ein Patient durch häufige «Ja, aber»-Sätze» aus, dass er auf der Stelle tritt ( «ja, Sie haben Recht, dass es besser wäre, nicht zu rauchen, aber ich denke, ich muss einfach ab und zu eine rauchen»).

Die vernebelnde Wirkung des Wortes «eigentlich» wurde bereits von einigen Dichtern und Denkern erkannt. Kurt Tucholsky soll 1928 formuliert haben: «Es gibt so viel Verhinderte ... Da sind die 〈Nur-Journalisten〉, die <eigentlich〉 Dichter sind, so große lyrische Dichter, dass Stefan George von Glück sagen kann; <eigentliche> Musiker gibt es zu Hunderttausenden, es ist ein Glück, dass uns die meisten erspart bleiben. Es kommt aber, wenn's soweit ist, gar nicht auf das Eigentliche an. «Eigentlich〉 haben die Richter ihn freisprechen wollen, aber dann haben sie ihn doch verurteilt ... und nun sitzt er im Gefängnis und kann mit dem Eigentlichen nicht viel beginnen. 〈Eigentlich〉sollte er Privatdozent werden, aber er ist dann doch in die Industrie gegangen ... Ein merkwürdiges Wechselspiel: mal ist das zugrunde liegende «Eigentliche〉 unwahr, und mal stimmt die Oberfläche nicht ... Weil aber keiner ganz er selbst ist, so bleibt immer ein kleines «Eigentlich〉 übrig, auf das er sich, bei Bedarf, zurückziehen kann. Denn was wollen sie eigentlich alle -? Proprement dit: das Glück.» [Meinke, 1998].
In «Der Kleine Prinz» lässt Saint-Exupery den Fuchs zum Kleinen Prinzen sagen: «L'essentiel est invisible pour les yeux» (zu deutsch: Das Eigentliche ist unsichtbar für die Augen). Der Religionskritiker Eugen Drewermann [2000] verwendete dies als Titel für eines seiner Bücher. Hier ist «eigentlich» im Sinne von «das Wesentliche» gemeint. Das Eigentliche kann für unsere Augen unsichtbar sein, doch wir können seinen tieferen Sinn erfassen. Theodor Adorno sagte schon im Jahr 1939: «Eigentlich kann man nichts mehr sagen. Die Tat ist die einzige Form, die der Theorie noch bleibt.» Danach hat er noch viele Bücher geschrieben und Vorlesungen und Vorträge gehalten. Den Aufruf zur Tat griffen später Studenten der 68er Generation auf. Adorno wollte andererseits den Philosophen Martin Heidegger am «Jargon der Eigentlichkeit» entlarven [Der Spiegel, 2003].

Der deutsche Lyriker Robert Gernhardt [Freund, 1997] schrieb ein Gedicht mit dem Titel «Eigentlich nicht»:

Das nennt man nicht eigentlich suchen,

wenn man schon weiß, wo was ist.

Das nennt man nicht eigentlich finden,

wenn man es gar nicht vermisst.

Das nennt man nicht eigentlich lieben, wenn man den Liebling erpresst.

Das nennt man nicht eigentlich halten, wenn man ihn fallen lässt.

«Eigentlich-Sätze» können Sinn verwischend, verwirrend wirken. Hypnotherapeuten [Freund, 2003; Prior, 2003] weisen darauf hin, dass «Eigentlich-Sätze» und andere vernebelnde Formulierungen des Patienten beim Therapeuten Trance induzierend wirken können; wenn der Therapeut dies nicht merkt, kann er schnell davon eingelullt werden und damit seine Gestaltungsmöglichkeit der Psychotherapie verlieren. Das Wort «eigentlich» hat einen Chamäleoncharakter indem man es einem Satz hinzufügt, wird dieser mehrdeutig weit entfernt von der neutestamentarischen Aussage «Deine Worte seien 〈ja ja - nein nein»». Der eindeutige Sinn einer

\begin{tabular}{ll}
\hline KARGER & @ 2003 S. Karger GmbH, Freiburg \\
Fax +49 7614520714 & Accessible online at: \\
$\begin{array}{l}\text { E-mail Information@Karger.de } \\
\text { www.karger.com }\end{array}$ & www.karger.com/ver
\end{tabular}


Aussage wird dadurch für den Therapeuten schwer fassbar. Viele in der Therapie verwendete «Eigentlich-Sätze» drücken eine Ambivalenz des Patienten aus. Der Therapeut tut gut daran, sich dafür zu sensibilisieren und damit Ambivalenzen und mangelnde Klarheit in der Festlegung eines Patienten zu erfassen. Ein «Eigentlich-Satz» muss erst hinsichtlich seines vom Patienten gemeinten Sinnes überprüft werden. Dies kann z.B. geschehen, durch die Umkehr des Eigentlich-Satzes. «Eigentlich will ich mit dem Trinken aufhören», wird dann zu: «Ich will nicht mit dem Trinken aufhören». Eine alternative Aussage des Eigentlich-Satzes kann hervortreten, wenn wir das «eigentlich» durch «im Grunde» ersetzen: «Im Grunde will ich mit dem Trinken aufhören». Dies kommt der ursprünglichen Bedeutung von «eigentlich» zwar näher, ist allerdings oft von unseren Patienten so nicht gemeint. Hilfreich kann nach einem «Eigentlich-Satz» die Nachfrage sein: «Und was wollen Sie uneigentlich?»

«Eigentlich wollte ich mir das Leben nehmen!» Was will der Patient damit wirklich ausdrücken? Dies kann von Fall zu Fall verschieden sein. Es kann gemeint sein: «Im Grunde bin ich froh, noch zu leben» oder «im Grunde möchte ich tot sein» oder «damals war ich so dumm, daran zu denken, mir das Leben zu nehmen, doch heute weiß ich, dass ich leben will». «Eigentlich wollte ich mir das Leben nehmen» spricht von der Vergangenheit und sagt nichts über die Gegenwart, nach der der Therapeut erst fragen muss.

Was meint der Patient, wenn er sagt: «Eigentlich halte ich nichts von einer Psychotherapie», aber doch zu einer Psychotherapiesitzung gekommen ist? Will er ausdrücken, dass er im Grunde nichts davon hält und nur aus Höflichkeit ein paar Sitzungen absolvieren wird, um dann «danke, nein» zu sagen? Oder ist es ein verstecktes Lob an den Therapeuten, der einen Patienten, der nichts von Psychotherapie hält, trotzdem in einer Therapie halten kann (cave!)? Was meint der Patient, der sagt: «Eigentlich habe ich schon alles probiert»? Warum sagt er nicht: «Ich kann mir nicht vorstellen, dass nach allem, was ich schon versucht habe, mir eine Psychotherapie helfen wird»? So lässt er dem Therapeuten ein Quäntchen Hoffnung, dass dieser vielleicht doch noch ein Wunder vollbringt. Warum kommt er überhaupt zu der Sitzung, wenn er sich nichts von einer weiteren Therapie verspricht? Was meint ein Patient, der sagt: «Eigentlich sollte mein Partner hier sein»? Meint er, der Partner solle dazukommen? Meint er, der Partner sei an allem schuld? Meint er, er selbst sei ganz gesund, nur der Partner müsse sich ändern?

Etymologisch stammt das heutige Wort «eigentlich» von dem altgermanischen und mittelhochdeutschen Wort «eigen»ab. «Eigen» ist das Adjektiv des früh verselbstständigten zweiten Partizips eines im Deutschen untergegangenen gemeingermanischen Verbs mit der Bedeutung «haben, besitzen» und bedeutet demnach «in Besitz genommen, besessen». Diese Bedeutung findet sich noch in dem Wort «leibeigen». Heute drückt es mehr die Zugehörigkeit aus (sein eigenes Haus) und steht auch umschreibend für selbst bzw. selbstständig (auf ei- genen Füßen). Dies drückt sich auch aus in Worten wie eigenhändig, -mächtig, -nützig, -sinnig. Das Wort «eigentlich» drückte ursprünglich «das (leib)eigene» aus, später «ausdrücklich, bestimmt» und heute «ursprünglich, wirklich, genau genommen». Die letzte Silbe des Wortes «eigentlich», -lich, stammt aus dem Mittelhochdeutschen und war ursprünglich ein selbstständiges Wort. Aus dem selben Stamm leitet sich das Wort Leiche ab (Körper, Gestalt). Als Grundwort in einer Wortzusammensetzung bedeutet es «die Gestalt habend», als Suffix drückt es zunächst eine wesensgemäße Eigenschaft bzw. Merkmale verschiedener Art aus. Somit ist die wörtliche Bedeutung von «eigentlich»: «die Gestalt des Wirklichen habend».

Grammatik, Pragmatik und Gesprächsanalyse sind Fachgebiete, die sich mit Worten wie «eigentlich» viel befasst haben [El Souessi, 2002, persönliche Mitteilung]. Nach den Einteilungen der wissenschaftlichen Grammatik ist das Wort «eigentlich» ein so genannter Partikel [Helbig und Buscha, 1992]. Andere Partikel sind: aber, auch, bloß, denn, doch, eben, etwa, halt, ja, mal, nur, schon, vielleicht und wohl. Jeder einzelne Partikel ist es wert, vom Therapeuten besonders beachtet zu werden. Partikel haben bestimmte Funktionen. Eine ihrer Funktionen ist eine illokutive. Das Hinzufügen eines Partikels hat eine Bedeutung. Die Frage «Was soll ich in der Therapie?» ist eindeutig. Die Bedeutung der Frage «Was soll ich eigentlich/bloß/ denn/überhaupt/wohl in der Therapie?» hängt unter anderem vom Kontext ab. Sie hängt von den Vor- und Nachsätzen ab («Ich habe schon so viele Therapien gemacht, warum soll ich denn eigentlich noch eine Therapie machen? Ich glaube das bringt alles nichts»). Der eigentliche Satz ist hier eingebettet in Sätze, aus denen sich die Bedeutung des «Eigentlich-Satzes» genauer ableiten lässt. «Eigentlich» ist ein Partikel, der anders als andere - nicht bei Ausrufe- und Aufforderungsintentionen des Sprechers, sondern in Aussage- und Fragesätzen (mit Frageintention oder als implizite Antwort) vorkommt: «Die Therapie ist eigentlich doch ganz hilfreich», «Was meinen Sie selbst eigentlich dazu?». Das Wort «eigentlich» kann betont oder nicht betont werden: «Eigentlich bin ich jetzt nicht weiter als am Anfang» bzw. «Ich weiß eigentlich immer noch nicht, worauf Sie hinauswollen». «Eigentlich» als Partikel kann einen neuen Aspekt ausdrücken, der meist einen wesentlichen Gedanken enthält, demgegenüber das bisher Gesagte vordergründig und oberflächlich erscheint (= im Grunde genommen, bei tieferer Überlegung): «Eigentlich habe ich mich schon verloren geglaubt» (auch wenn ich bei genauerer Betrachtung sehe, dass dies voreilig war). Oder: «Was denken Sie eigentlich?». Oder: «Was denken Sie eigentlich wirklich?» (im Inneren Ihres Wesens).

Von den vielen verschiedenen Bedeutungen, die «eigentlich» haben kann, sind zwei für den Therapeuten besonders wichtig: 1. «Eigentlich» im Sinne des Ursprünglichen, des Wirklichen, des Genau-Genommenen, des Im-Grunde-und-bei-tiefererÜberlegung-Gemeinten sowie 2. im Sinne einer Vernebelung, Bedeutung kaschierenden, vagen, sinnverwischenden, verwir- 
renden Bedeutung. Die Bedeutung kaschierende Funktion des Wortes «eigentlich», dürfte im Rahmen der Psychotherapie sehr häufig sein.

Für ein Verständnis der jeweiligen Bedeutung des Wortes «eigentlich» ist, wie schon erwähnt, der Kontext relevant. Häufig können wir die jeweilige Bedeutung des Wortes «eigentlich» nur einschätzen und verstehen, wenn wir genau auf den verbalen und nonverbalen Kontext achten, in den es eingebettet ist. So können relevant sein: 1 . Vorausgegangene und nachfolgende Worte und Sätze, 2. die Betonung der Worte und 3. begleitende Blicke, Mimik und Gesten. Auch wenn das Wort «eigentlich» oft zu vernebeln scheint, kann es uns in der Kommunikation helfen, die Dinge noch präziser, als durch klare Worte auszudrücken. Partikel helfen uns, Nuancen auszudrücken und diese sind in der Psychotherapie besonders wichtig. Um sie zu verstehen, ist es somit wichtig, auf den gesamten Kontext $\mathrm{zu}$ achten, in den das Wort «eigentlich» eingebettet ist.

Dies alles ist doch eigentlich nicht der Rede wert! - Oder?

Manfred M. Fichter, Prien

\section{Literatur}

Drewermann E: Das Eigentliche ist unsichtbar. Der Kleine Prinz tiefenpsychologisch gedeutet. Freiburg, Herder, 2000.

Duden Etymologie: Das Herkunftswörterbuch der deutschen Sprache. Duden Bd. 7. Mannheim, Bibliographisches Institut, 1963.

Der Spiegel: Narziss und Nilpferdkönig. 2003;34:143.

El-Souessi K: Persönliche Mitteilung, 2002.

Freund U: Auf des Messers Schneide «Eigentlich überflüssig». M.E.G.a.Phon, Informationsblatt der Milton Erickson Gesellschaft 1997;26:4-5

Helbig G, Buscha J: Deutsche Grammatik. München, Langenscheidt, 1992.

Meinke C: Leserbrief. M.E.G.a.Phon, Informationsblatt der Milton Erickson Gesellschaft 1998;27:4-5.

Prior M: MiniMax-Interventionen. Heidelberg, Carl Auer, 2003. 